\title{
SOME CANONICAL FORMS AND ASSOCIATED CANONICAL EXPANSIONS IN PROJECTIVE DIFFERENTIAL GEOMETRY*
}

BY E. B. STOUFFER

1. Introduction. A simplification of the methods of approach to any branch of mathematics is always very desirable. This is particularly true in a geometry, where extensive analytical machinery must be set up before geometric results can be obtained. This paper is a contribution to the simplification of Wilczynski's methods of attack upon plane and space curves in projective differential geometry. $\dagger$

A canonical form for the fundamental differential equation associated with the curve is determined. It leads at once to a complete and independent system of invariants and covariants in their canonical form. The determination of the corresponding system in the general form involves only simple substitutions. An associated canonical expansion for the equation or equations of the curve is obtained by very direct methods and the geometrical significance of the corresponding triangle or tetrahedron of reference becomes easily evident. Because of the method of their derivation, the fundamental invariants and covariants obtained are those which have an immediately evident geometrical significance.

The methods here employed may be applied to surfaces, both curved and ruled in ordinary space and may also be extended to geometry in hyperspace. The resulting simplifications are in some cases quite remarkable. These results will be presented in later papers.

* Part of a paper read upon invitation of the Program Committee at a meeting of the Southwestern Section of the Society, St. Louis, November 26, 1927.

† See Wilczynski, Projective Differential Geometry of Curves and Ruled Surfaces, Chapters 2, 3 and 13. 
In a series of papers* published in recent years, Sannia has made a study of plane and space curves by methods related somewhat to those used in this paper. He employs the absolute calculus but the results obtained below show that its use is unnecessary in order to gain real simplicity. Moreover, the methods of Sannia by no means extend themselves so naturally and so simply to surfaces in ordinary space and to hyperspace.

2. Plane Curves. It is well known that all the projective differential properties of plane curves may be studied by means of a single differential equation of the form

$$
y^{\prime \prime \prime}+3 p_{1} y^{\prime \prime}+3 p_{2} y^{\prime}+p_{3} y=0,
$$

where differentiation is with respect to the independent variable $x$ and where $p_{i}$ are functions of $x$. If the homogeneous coordinates of a curve $C$ are three functions $y_{i}(i=1,2,3)$ of $x$ then the coefficients $p_{i}$ may be so determined that $y_{i}$ form a fundamental set of solutions of (A), provided merely that $C$ is not a straight line. Moreover, any other set of fundamental solutions $z_{i}(i=1,2,3)$ of (A) may be expressed in the form

$$
z_{i}=\sum_{j=1}^{3} c_{i j} y_{j}, \quad(i=1,2,3), \quad\left|c_{i j}\right| \neq 0 .
$$

Consequently all the curves determined by (A) in this way are projective transformations of $C$.

However, the parametric representation of $C$ is not unique. A transformation of the independent variable of the form

$$
\bar{x}=\phi(x),
$$

and of the dependent variable of the form

$$
y=\lambda \bar{y},
$$

where $\phi$ and $\lambda$ are arbitrary functions of $x$, will not change the curve. Moreover, the form of (A) will not be changed

* Rendiconti dei Lincei, (5), vol. $31,1^{\circ}$ sem., pp. 450-454 and pp. 503506; (5), vol. $31,2^{\circ}$ sem., pp. 17-19 and pp. 432-434; Annali di Matematica Pura ed Applicata, (4), vol. 1, pp. 1-18; (4), vol. 3, pp. 1-25. 
by the above transformations but it will be converted into an equation with new coefficients. A function of the new coefficients and the new dependent variable and their derivatives which is equal, except for a factor, to the same function of the original coefficients and the original dependent variable and their derivatives is said to be a relative covariant. If the function does not contain the dependent variable or its derivatives, it is said to be an invariant.

The effect of the transformations is important. From (1) we have

$$
\left\{\begin{array}{c}
y^{\prime}=\frac{d y}{d \bar{x}} \phi^{\prime}, y^{\prime \prime}=\frac{d y^{2}}{d \bar{x}^{2}}\left(\phi^{\prime}\right)^{2}+\frac{d y}{d \bar{x}} \phi^{\prime \prime}, \\
y^{\prime \prime \prime}=\frac{d^{3} y}{d \bar{x}^{3}}\left(\phi^{\prime}\right)^{3}+3 \frac{d^{2} y}{d \bar{x}^{2}} \phi^{\prime} \phi^{\prime \prime}+\frac{d y}{d \bar{x}} \phi^{\prime \prime \prime},
\end{array}\right.
$$

whence, by substitution into (A), we obtain a new equation

where

$$
\frac{d^{3} y}{d \bar{x}^{3}}+3 \bar{p}_{1} \frac{d^{2} y}{d \bar{x}^{2}}+3 \bar{p}_{2} \frac{d y}{d \bar{x}}+\bar{p}_{3} y=0
$$

$\left\{\begin{array}{l}\bar{p}_{1}=\frac{1}{\phi^{\prime}}\left(p_{1}+\frac{\phi^{\prime \prime}}{\phi^{\prime}}\right), \\ \bar{p}_{2}=\frac{1}{\left(\phi^{\prime}\right)^{2}}\left(p_{2}+p_{1} \frac{\phi^{\prime \prime}}{\phi^{\prime}}+\frac{\phi^{\prime \prime \prime}}{3 \phi^{\prime}}\right), \quad \bar{p}_{3}=\frac{1}{\left(\phi^{\prime}\right)^{3}} p_{3} .\end{array}\right.$

Likewise the transformation (2) gives

$$
\left\{\begin{array}{l}
y^{\prime}=\bar{\lambda} \bar{y}^{\prime}+\bar{\lambda}^{\prime} \bar{y}, y^{\prime \prime}=\bar{\lambda} \bar{y}^{\prime \prime}+2 \bar{\lambda}^{\prime} \bar{y}^{\prime}+\bar{\lambda}^{\prime \prime} \bar{y}, \\
y^{\prime \prime \prime}=\bar{\lambda} \bar{y}^{\prime \prime \prime}+3 \bar{\lambda}^{\prime} \bar{y}^{\prime \prime}+3 \bar{\lambda}^{\prime \prime} \bar{y}^{\prime}+\bar{\lambda}^{\prime \prime \prime} \bar{y},
\end{array}\right.
$$

whence by substitution into (4) we obtain a new equation in $\bar{y}$ whose coefficients $\bar{P}_{i}$ are expressed by the equations

$$
\left\{\begin{array}{l}
\bar{\lambda} \bar{P}_{1}=\bar{\lambda} \bar{p}_{1}+\bar{\lambda}^{\prime}, \bar{\lambda} \bar{P}_{2}=\bar{\lambda} \bar{p}_{2}+2 \bar{\lambda}^{\prime} \bar{p}_{1}+\bar{\lambda}^{\prime \prime}, \\
\bar{\lambda} \bar{P}_{3}=\bar{\lambda} \bar{p}_{3}+3 \bar{\lambda}^{\prime} \bar{p}_{2}+3 \bar{\lambda}^{\prime \prime} \bar{p}_{1}+\bar{\lambda}^{\prime \prime \prime}
\end{array}\right.
$$

where differentiation is with respect to $\bar{x}$. 
All of the above is well known theory.* However, for the purposes of the present paper it is important to notice that equations (3), (5), (6), (7) show the following significant fact. If by means of (1) and (2) equation (A) is transformed into a canonical form which is preserved if and only if

$$
\phi^{\prime \prime}=0, \frac{d \bar{\lambda}}{d \bar{x}}=0,
$$

then the new variable $\bar{y}$ and the new coefficients $\bar{P}_{i}$ and their derivatives are determined except for multiplication by certain factors.

The resulting variable $\bar{y}$ and its derivatives will each be the canonical form of a relative covariant and must each determine a unique point in the plane. Likewise, the resulting coefficients $\bar{P}_{i}$ and their derivatives must each be the canonical form of a relative invariant. We shall see later that the determination of the expressions for these invariants and covariants in terms of the coefficients and variables of (A) will involve only simple substitutions by means of equations (3), (5), (6), (7).

The determination of the transformations which give a canonical form to our equations may be made in many ways and many different canonical forms may be obtained. It is evident that each such canonical form must lead directly to an equation for the curve in the form of an expansion of one non-homogeneous coordinate in terms of the other. Since such an expansion determines the geometrical properties of the curve, we naturally desire that the canonical form of (A) be such as to simplify the process of obtaining these properties from the associated expansion.

We now assume that our fundamental differential equation has been transformed into such a canonical form with coefficients $\bar{P}_{i}$ and variables $\dagger \bar{x}$ and $\bar{y}$ and proceed to calculate

* See Wilczynski, loc. cit., Chapter III.

$\dagger$ In the remainder of this paper a bar above a letter will indicate that it represents a function of $\bar{x}$ and that differentiation of the function is with respect to $\bar{x}$. 
the corresponding canonical expansion. It is an important fact that we can proceed just as if the conditions to be imposed were actually known.

Let $\bar{y}_{i}(i=1,2,3)$ be the coordinates of a regular point* $y$ on $C$. We may assume without loss of generality that this point $y$ is given by the value $\bar{x}=0$ of the parameter. Then by the general theory of differential equations the coordinates $\bar{Y}_{i}$ of any point $\bar{Y}$ on the curve in the neighborhood of $\bar{y}$ may be expressed as a power series in $\bar{x}$ of the form

$$
\bar{Y}=\bar{y}(0)+\bar{y}^{\prime}(0) \bar{x}+\bar{y}^{\prime \prime}(0) \frac{\bar{x}^{2}}{2}+\bar{y}^{\prime \prime \prime}(0) \frac{\bar{x}^{3}}{3 !}+\cdots .
$$

When we start to substitute for $\bar{y}^{\prime \prime \prime}(0), \bar{y}^{\mathrm{iv}}(0), \cdots$ by means of the assumed canonical form of our differential equation, we see at once that a great simplification will take place if $\bar{P}_{1}=0$. The first of equations (7) shows that we can make $\bar{P}_{1}=0$ by choosing $\bar{\lambda}$ to satisfy the equation $\bar{\lambda} \bar{p}_{1}+\bar{\lambda}^{\prime}=0$. Moreover, equations (5) and (7) show that the condition $\bar{P}_{1}=0$ will be maintained if $(8)$ is satisfied. We shall therefore assume that $\bar{P}_{1}=0$ is one of the conditions imposed in order to give us our canonical form. Thus the canonical form is of the nature

$$
\bar{y}^{\prime \prime \prime}+3 \bar{P}_{2} \bar{y}^{\prime}+\bar{P}_{3} \bar{y}=0,
$$

where the coefficients are determined by the substitution of $\bar{\lambda}^{\prime}=-\bar{\lambda} \bar{p}_{1}$ into (7) and have the form

$$
\left\{\begin{array}{l}
\bar{P}_{2}=\bar{p}_{2}-\bar{p}_{1}^{\prime}-\bar{p}_{1}^{2}, \\
\bar{P}_{3}=\bar{p}_{3}-3 \bar{p}_{1} \bar{p}_{2}+2 \bar{p}_{1}^{3}-\bar{p}_{1}^{\prime \prime} .
\end{array}\right.
$$

The substitution from (5) into (10) gives

* Throughout this paper a point with coordinates $\alpha_{i}(i=1,2,3)$ in the plane or with coordinates $\alpha_{i}(i=1,2,3,4)$ in space will be denoted simply by $\alpha$ when no confusion can arise. 


$$
\left\{\begin{array}{l}
\bar{P}_{2}=\frac{1}{\left(\phi^{\prime}\right)^{2}}\left(P_{2}-\frac{2}{3} \psi^{\prime}+\frac{1}{3} \psi^{2}\right) \\
\bar{P}_{3}=\frac{1}{\left(\phi^{\prime}\right)^{3}}\left(P_{3}-3 \psi P_{2}+3 \psi \psi^{\prime}-\psi^{3}-\psi^{\prime \prime}\right),
\end{array}\right.
$$

where $\psi=\phi^{\prime \prime} / \phi^{\prime}$ and where $P_{2}$ and $P_{3}$ are the same functions of $p_{i}$ as are $\bar{P}_{2}$ and $\bar{P}_{3}$ of $\bar{p}_{i}$. The expressions for the derivatives of $\bar{P}_{2}$ and $\bar{P}_{3}$ are obtained from (11) by simple differentiation. The particular value of $\psi$ which gives the canonical form is as yet undetermined.

Substitution from (9) in to the above expansion now gives*

$$
\left\{\begin{aligned}
\bar{Y}= & \bar{y}+\bar{y}^{\prime} \bar{x}+\bar{y}^{\prime \prime} \frac{\bar{x}^{2}}{2}-\left(3 \bar{P}_{2} \bar{y}^{\prime}+\bar{P}_{3} \bar{y}\right) \frac{\bar{x}^{3}}{3 !} \\
& -\left[3 \bar{P}_{2} \bar{y}^{\prime \prime}+\left(3 \bar{P}_{2}^{\prime}+\bar{P}_{3}\right) \bar{y}^{\prime}+\bar{P}_{3}^{\prime} \bar{y}\right] \frac{\bar{x}^{4}}{4 !} \\
& -\left[\left(6 \bar{P}_{2}^{\prime}+\bar{P}_{3}\right) \bar{y}^{\prime \prime}+\left(2 \bar{P}_{3}^{\prime}+3 \bar{P}_{2}^{\prime \prime}-9 \bar{P}_{2}^{2}\right) \bar{y}^{\prime}\right. \\
& \left.+\left(\bar{P}_{3}^{\prime \prime}-3 \bar{P}_{2} \bar{P}_{3}\right) \bar{y}\right] \frac{\bar{x}^{5}}{5 !} \\
& -\left[\left(9 \bar{P}_{2}^{\prime \prime}+3 \bar{P}_{3}^{\prime}-9 \bar{P}_{2}^{2}\right) \bar{y}^{\prime \prime}\right. \\
& +\left(3 \bar{P}_{3}^{\prime \prime}+3 \bar{P}_{2}^{\prime \prime}-36 \bar{P}_{2} \bar{P}_{2}^{\prime}-6 \bar{P}_{2} \bar{P}_{3}\right) \bar{y}^{\prime} \\
& \left.+\left(\bar{P}_{3}^{\prime \prime}-9 \bar{P}_{2}^{\prime} \bar{P}_{3}-\bar{P}_{3}^{2}-3 \bar{P}_{2} \bar{P}_{3}^{\prime}\right) \bar{y}\right] \frac{\bar{x}^{6}}{6 !}+\cdots
\end{aligned}\right.
$$

Since we are assuming that we have a canonical form, $\bar{y}_{i}, \bar{y}_{i}^{\prime}, \bar{y}_{i}^{\prime \prime}$ are the homogeneous coordinates of three fixed points not on a straight line. Consequently, the coordinates of any point in the plane may be expressed in the form $x_{1} \bar{y}_{i}+x_{2} \bar{y}_{i}^{\prime}+x_{3} \bar{y}_{i}^{\prime \prime}$. It follows that the coordinates of the point may be taken to be $\left(x_{1}, x_{2}, x_{3}\right)$. The coordinates $x_{i}$ of $\bar{Y}$ in this new coordinate system may be read directly from equation (12) and have the form of expansions in powers of $\bar{x}$.

* It is to be understood that in the coefficients of this expansion and those that follow $\bar{x}=0$. 
If we now pass to non-homogeneous coordinates we obtain the following results:

$$
\left\{\begin{aligned}
\xi= & \frac{x_{2}}{x_{1}}=\bar{x}-\frac{1}{2} \bar{P}_{2} \bar{x}^{3}-\frac{1}{8}\left(\bar{P}_{2}^{\prime}-\bar{P}_{3}\right) \bar{x}^{4} \\
& +\left(3 \bar{P}_{3}^{\prime}-3 \bar{P}_{2}^{\prime \prime}+9 \bar{P}_{2}\right) \frac{\bar{x}^{5}}{5 !}+\cdots, \\
\eta= & \frac{x_{3}}{x_{1}}=\frac{\bar{x}^{2}}{2}-\frac{1}{8} \bar{P}_{2} \bar{x}^{4}-\left(6 \bar{P}_{2}^{\prime}-9 \bar{P}_{3}\right) \frac{\bar{x}^{5}}{5 !} \\
& +\left(9 \bar{P}_{2}^{2}+12 \bar{P}_{3}^{\prime}-9 \bar{P}_{2}^{\prime \prime}\right) \frac{\bar{x}^{6}}{6 !}+\cdots
\end{aligned}\right.
$$

The elimination of $\bar{x}$ from these equations gives us the desired expansion

$$
\left\{\begin{aligned}
\eta= & \frac{1}{2} \xi^{2}+\frac{3}{8} \bar{P}_{2} \xi^{4}+\frac{1}{40}\left(3 \bar{P}_{2}^{\prime}-2 \bar{P}_{3}\right) \xi^{5} \\
& +\left(9 \bar{P}_{2}^{\prime \prime}-6 \bar{P}_{3}^{\prime}+405 \bar{P}_{2}^{2}\right) \frac{\xi^{6}}{6 !}+\cdots
\end{aligned}\right.
$$

We return now to the problem of the determination of our canonical form. It is easy to see from equations (11) that the term $3 \bar{P}_{2}^{\prime}-2 \bar{P}_{3}=\bar{\theta}_{3}$ in the coefficient of $\xi^{5}$ in (14) is such that

$$
\bar{\theta}_{3}=\frac{1}{\left(\phi^{\prime}\right)^{3}}\left(3 P_{2}^{\prime}-2 P_{3}\right)=\frac{1}{\left(\phi^{\prime}\right)^{3}} \theta_{3} .
$$

Consequently, assuming that $\theta_{3}$ does not vanish identically,* we can make $\bar{\theta}_{3}=1$ by selecting $\left(\phi^{\prime}\right)^{3}=\theta_{3}$. It is evident that the two conditions $\bar{\theta}_{3}=1$ and $\bar{P}_{1}=0$ are preserved only if $\phi^{\prime \prime}=0, \bar{\lambda}^{\prime}=0$. We then have for a canonical form of (A)

$$
\frac{d^{3} \bar{y}}{d \bar{x}^{3}}+3 \bar{P}_{2} \frac{d \bar{y}}{d \bar{x}}+\bar{P}_{3} \bar{y}=0,
$$

with $\bar{\theta}_{3}=1$, and for the associated canonical expansion

* It is easily seen that the identical vanishing of $\theta_{3}$ is the condition that $C$ be a conic. The projective differential properties of conics are of no interest. 


$$
\eta=\frac{1}{2} \xi^{2}+\frac{3}{8} \bar{P}_{2} \xi^{4}+\frac{1}{40} \xi^{5}+\frac{9}{16} \bar{P}_{2} \xi^{6}+\cdots
$$

The coefficients $\bar{P}_{2}$ and $\bar{P}_{3}$ and their derivatives are all canonical forms of relative invariants. In order to obtain their general form it is only necessary to substitute the value of $\psi$ obtained from $\left(\phi^{\prime}\right)^{3}=\theta_{3}$ into equations (1) and their derivatives. That the expressions thus obtained are relative invariants becomes evident if we take any two equations of form (A) which are equivalent under (1) and (2) and reduce each of them to our canonical form. The coefficients thus obtained can differ only by a factor and consequently the expressions for these coefficients in terms of the original coefficients will have the same form and can differ only by a factor.

That the invariants thus obtained together with $\theta_{3}$ form a complete system is evident. In fact, since $3 \bar{P}_{2}^{\prime}-2 \bar{P}_{3}=1$ we actually need only the invariants arising from $\bar{P}_{2}$ and its derivatives together with $\theta_{3}$ in order to have a complete system. That all the invariants in the latter complete system are independent is also evident.

Exactly the same type of argument shows that $\bar{y}, \bar{y}^{\prime}, \bar{y}^{\prime \prime}$ are the canonical forms of relative covariants which may be expressed in terms of the original coefficients and variables by means of (3), (5), (6), (7) with the now known values of $\phi^{\prime \prime}$ and $\bar{\lambda}^{\prime}$ substituted. That the covariants thus obtained are independent is obvious. That they form with the complete system of invariants a complete system of covariants follows from the fact that the higher derivatives of $\tilde{y}$ than the second can be replaced by means of equation (15).

We have thus obtained a complete and independent system of invariants and covariants from our canonical form by a process which involves nothing more difficult than direct substitutions.

The three fundamental covariants $\bar{y}, \bar{y}^{\prime}, \bar{y}^{\prime \prime}$ determine precisely the three vertices of the tetrahedron of reference. The geometric determination of these vertices is not 
difficult and gives results obtained by Wilczynski and Sannia. Equation (16) gives, by the method of undetermined coefficients, for the equation of the conic osculating $C$ at $\bar{y}$

$$
2 x_{3}\left(x_{1}-(3 / 2) \bar{P}_{2} x_{3}\right)-x_{2}{ }^{2}=0,
$$

and for the equation of the cubic osculating $C$ at $\bar{y}$ and having a node at that point

$$
10 x_{2} x_{3}\left(x_{1}-(3 / 2) \bar{P}_{2} x_{3}\right)-5 x_{2}{ }^{3}-2 x_{3}{ }^{3}=0 .
$$

The tangent at $(1,0,0)$, which is the point $\bar{y}$, to one branch of the cubic (18) is the line $x_{2}=0$ and the pole of this line with respect to the conic $(17)$ is $(0,1,0)$. Since $(0,1,0)$ is the point $\bar{y}^{\prime}$ and $(0,0,1)$ is the point $\bar{y}^{\prime \prime}$, it is evident that the latter vertex is the point of intersection of the line $x_{2}=0$ with the tangent at $\bar{y}^{\prime}$ to the curve generated by $\bar{y}^{\prime}$.

One of the tangents from $(0,1,0)$ to the conic $(17)$ is $x_{3}=0$. The other tangent meets the cubic (18) in three distinct points, any one of which points may be used to determine the unit point of our system of coordinates.

Equation (15) shows at once the geometrical significance of the vanishing of the two fundamental invariants $\bar{P}_{2}$ and $\bar{P}_{3}$. If $\bar{P}_{3}=0$ the curve generated by the point $\bar{y}^{\prime}$ is a plane curve. If $\bar{P}_{2}=0$ the tangent at $\bar{y}^{\prime \prime}$ to the curve generated by that point passes through $\bar{y}$, that is, the curve generated by $\bar{y}^{\prime \prime}$ is the envelope of the line $x_{2}=0$.

It is interesting to note at this point that essentially the canonical expansion used by Wilczynski is obtained if the vertex $(0,0,1)$ is transformed to the second point of intersection of the line $x_{2}=0$ with the osculating conic.

All the machinery necessary for the study of the projective differential properties of plane curves has now been set up.

3. Space Curves. The projective differential properties of space curves can be studied by means of a differential equation of the fourth order of the form

$$
y^{\text {(iv) }}+4 p_{1} y^{\prime \prime \prime}+6 p_{2} y^{\prime \prime}+4 p_{3} y^{\prime}+p_{4} y=0 .
$$


The transformations of the variables which are permitted are exactly (1) and (2). In fact the methods employed and the equations derived in order to obtain the canonical expansion are obvious extensions of the methods and equations for plane curves. In place of equation (14) above we obtain in this case two equations

$$
\left\{\begin{array}{l}
\eta=\frac{1}{2} \xi^{2}-\frac{1}{4} \bar{P} \xi^{4}-2\left(3 \bar{P}_{2}^{\prime}-8 \bar{P}_{3}\right) \frac{\xi^{5}}{5 !} \\
-\left(6 \bar{P}_{2}^{\prime \prime}-16 \bar{P}_{3}^{\prime}+10 \bar{P}_{4}-36 \bar{P}_{2}^{2}\right) \frac{\xi^{6}}{6 !}+\cdots \\
\zeta=\frac{1}{6} \xi^{3}-\frac{1}{20} \bar{P}_{2} \xi^{5}-\frac{1}{180}\left(3 \bar{P}_{2}^{\prime}-14 \bar{P}_{3}\right) \xi^{6}+\cdots
\end{array}\right.
$$

In these equations $\bar{P}_{2}, \bar{P}_{3}, \bar{P}_{4}$ are the coefficients of the canonical form of equation (B) concerning which we know as yet only that $\bar{P}_{1}=\bar{p}_{2}-\bar{p}_{1}^{\prime}-\bar{p}_{1}^{2}=0$. The condition which shall be imposed to produce $\bar{p}_{i}$ from $p_{i}$ remains to be determined. In the above equations

$$
\xi=\frac{x_{2}}{x_{1}}, \eta=\frac{x_{3}}{x_{1}}, \zeta=\frac{x_{4}}{x_{1}},
$$

where the homogeneous coordinates $\left(x_{1}, x_{2}, x_{3}, x_{4}\right)$ of a point in space follow from the coordinates of the point expressed in the form

$$
x_{1} \bar{y}_{i}+x_{2} \bar{y}_{i}^{\prime}+x_{3} \bar{y}_{i}^{\prime \prime}+x_{4} \bar{y}_{i}^{\prime \prime \prime} .
$$

Equations (19) do not indicate directly the conditions to be imposed in order to obtain a canonical form. In order to link our canonical form with the geometry we shall first calculate the osculating cubic, the simplest osculating curve other than the tangent line. Since a space cubic is determined by six points, the canonical expansion for the cubic osculating $C$ at $\bar{y}$ must agree with (19) up to and including the fifth powers of $\xi$.

It is easily seen that the parametric representation of the osculating cubic can be put into the form 


$$
\left\{\begin{array}{l}
x_{1}=1+a_{1} t+b_{1} t^{2}+c_{1} t^{3}, \\
x_{2}=t+b_{2} t^{2}+c_{2} t^{3}, \\
x_{3}=b_{3} t^{2} \\
x_{4}=c_{4} t^{3} .
\end{array}\right.
$$

From these equations the expansions for $\eta=x_{3} / x_{1}$ and $\zeta=x_{4} / x_{1}$ in terms of $\xi=x_{2} / x_{1}$ are easily obtained. If the coefficients of the necessary powers of $\xi$ are equated to the corresponding powers in (19), we obtain for the parametric equations of the osculating cubic

$$
\left\{\begin{array}{l}
x_{1}=1+\frac{9}{10} \bar{P}_{2} t^{2}-\frac{1}{30}\left(3 \bar{P}_{2}^{\prime}-8 \bar{P}_{3}\right) t^{3}, \\
x_{2}=t+\frac{7}{10} \bar{P}_{2} t^{3}, \quad x_{3}=\frac{1}{2} t^{2}, \quad x_{4}=\frac{1}{6} \cdot t^{3},
\end{array}\right.
$$

and for the corresponding expansions for the cubic,

$$
\begin{aligned}
& \eta=\frac{1}{2} \xi^{2}-\frac{1}{4} \bar{P}_{2} \xi^{4}-2\left(3 \bar{P}_{2}^{\prime}-8 \bar{P}_{3}\right) \frac{\xi^{5}}{5 !}+\frac{1}{200} \bar{P}_{2}^{2} \xi^{6}+\cdots \\
& \zeta=\frac{1}{6} \xi^{3}-\frac{1}{20} \bar{P}_{2} \xi^{5}-\frac{1}{90}\left(3 \bar{P}_{2}^{\prime}-8 \bar{P}_{3}\right) \xi^{6}+\cdots
\end{aligned}
$$

It now becomes evident that the projections of $C$ and the osculating cubic from $(0,0,0,1)$, or $\bar{y}^{\prime \prime \prime}$, upon the osculating plane $x_{4}=0$ will have contact of the sixth order if and only if the coefficients of $\xi^{6}$ in the first equation of (19) and the first equation of (22) are equal, i.e., if and only if

$$
\bar{M}=15 \bar{P}_{2}^{\prime \prime}-40 \bar{P}_{3}^{\prime}+25 \bar{P}_{4}-81 \bar{P}_{2}^{2}=0 .
$$

But the equations for the space curve corresponding to (11) for the plane curve show that

$$
\bar{M}=\frac{1}{\left(\phi^{\prime}\right)^{4}}\left(M+15 \psi \theta_{3}\right),
$$

where*

* The expressions for $\bar{P}_{i}$ in terms of $\bar{p}_{i}$ are obtained by the substitution of $\bar{\lambda}^{\prime}=-\bar{\lambda} \bar{p}_{1}$ into the equations for the space curve corresponding to $(7)$ 


$$
M=15 P_{2}^{\prime \prime}-40 P_{3}^{\prime}+25 P_{4}-81 P_{2}{ }^{2}, \theta_{3}=3 P_{2}^{\prime}-2 P_{3} .
$$

It follows at once that, if $\theta_{3} \neq 0$, we can make $\bar{M}=0$. The conditions $\bar{M}=0$ and $\bar{P}_{1}=0$ determine our canonical form.

The coefficients $\bar{P}_{2}, \bar{P}_{3}, \bar{P}_{4}$ and their derivatives are the canonical form of relative invariants. There exist, of course, the relations obtained from $\bar{M}=0$ and from the differentiation of this equation. The expressions for the invariants in terms of the original coefficients again involve only simple substitutions, use being made of the now known values of $\psi$ and $\bar{\lambda}^{\prime}$. The questions of the completeness and of the independence of the system are answered precisely as for plane curves.

The expressions $\bar{y}, \bar{y}^{\prime}, \bar{y}^{\prime \prime}, \bar{y}^{\prime \prime \prime}$ are canonical covariants which may be expressed in terms of the original coefficients and variables by direct substitutions. The covariants thus obtained, together with a complete system of invariants, give a complete system of covariants.

The vertices of the tetrahedron of reference are given by the four fundamental covariants $\bar{y}, \bar{y}^{\prime}, \bar{y}^{\prime \prime}, \bar{y}^{\prime \prime \prime}$. If $\theta_{3} \neq 0$, the plane $x_{3}=0$ is evidently the principal plane* of $C$ and its osculating cubic. The osculating cubic meets $x_{3}=0$ in $(1,0,0,0)$ and also in the point $\left(3 \bar{P}_{2}^{\prime}-8 \bar{P}_{3},-21 \bar{P}_{2}\right.$, $0,-5)$. The osculating plane to the cubic at this point intersects the tangent to $C$ at $\bar{y}$ in the point $(0,1,0,0)$. The totality of osculating planes to the cubic intersects $x_{4}=0$ in lines which envelope a conic, called the osculating conic, whose equation is

$$
72 \bar{P}_{2} x_{3}{ }^{2}-40 x_{1} x_{3}+15 x_{2}^{2}=0, x_{4}=0 .
$$

The polar of $(0,1,0,0)$ with respect to this conic is the line $x_{2}=0, x_{4}=0$. The tangent at $(0,1,0,0)$ to the curve generated by $\bar{y}^{\prime}$ intersects $x_{2}=0, x_{4}=0$ in $\bar{y}^{\prime \prime}$ which is $(0,0,1,0)$, and

for the plane curve. The equations can be found in Chapter 13 of Wilczynski's book. The expressions for $P_{i}$ in terms of $p_{i}$ are of the same form as those for $\bar{P}_{i}$ in terms of $\bar{p}_{i}$.

* Halphen, Journal de l'Ecole Polytechnique, vol. 28, p. 25. 
the tangent at this point to the curve generated by $\bar{y}^{\prime \prime}$ intersects $x_{3}=0$ in $\bar{y}^{\prime \prime \prime}$ which is $(0,0,0,1)$. The vertices of the tetrahedron of reference are thus completely determined geometrically. These are essentially the results obtained by Wilczynski and Sannia for the location of the vertices.

The canonical form of (B),

$$
\bar{y}^{(\text {iv })}+6 \bar{P}_{2} \bar{y}^{\prime \prime}+4 \bar{P}_{3} \bar{y}^{\prime}+\bar{P}_{4} \bar{y}=0
$$

shows at once the geometrical significance of the vanishing of the fundamental invariants $\bar{P}_{2}, \bar{P}_{3}, \bar{P}_{4}$. In fact, the curve generated by $\bar{y}^{\prime}$ is plane if and only if $\bar{P}_{4}=0$, and the tangent at $\bar{y}^{\prime \prime \prime}$ to the curve generated by this point intersects the line joining $\bar{y}$ and $\bar{y}^{\prime \prime}$ if and only if $\bar{P}_{3}=0$ and the line joining $\bar{y}$ and $\bar{y}^{\prime}$ if and only if $\bar{P}_{2}=0$.

It is easy to make a transformation of coordinates which gives the canonical expansion used by Wilczynski. It is only necessary to change the vertex $(0,0,1,0)$ in to the point where the line $x_{2}=0, x_{4}=0$ intersects the osculating conic and the vertex $(0,0,0,1)$ in to the point where the osculating cubic intersects $x_{3}=0$.

If $\theta_{3}=0$, the principal plane of $C$ and its osculating cubic is the osculating plane. This fact becomes evident if we observe that the coefficients of $\xi^{6}$ are equal in the second expansions of (19) and (22) if $\theta_{3}=0$. In this case it is easy to verify that the tangent plane at $\bar{y}$ to the quadric osculating $C$ at $\bar{y}$ is the plane $x_{3}=0$ if

$$
\bar{\theta}_{4}=\bar{P}_{4}-2 \bar{P}_{3}^{\prime}+(6 / 5) \bar{P}_{2}^{\prime \prime}-(81 / 25) \bar{P}_{2}{ }^{2}=1 .
$$

Since $\bar{\theta}_{4}=\theta_{4} /\left(\phi^{\prime}\right)^{4}$, we can make $\bar{\theta}_{4}=1$ by choosing $\left(\phi^{\prime}\right)^{4}=\theta_{4}$, if $\theta_{4} \neq 0$. A canonical form for (A) is thus determined in this case and associated with it, just as above, we have two expansions, and a geometrically determined tetrahedron of eference.

If both $\theta_{3}$ and $\theta_{4}$ vanish, the curve $C$ is a cubic and has no projective differential properties of interest.

The University of Kansas 